\title{
Compressive Sensing Based Data Collection in Wireless Sensor Networks
}

\author{
Liu Yuhong ${ }^{1, a}$ and Fu Jie ${ }^{1, b}$ \\ ${ }^{1}$ School of Electronic and Information Engineering, Lanzhou Jiaotong University, Lanzhou, 730070, \\ China \\ aliuyuhong518@163.com, b290067931@qq.com
}

Keywords: Wireless Sensor Networks, Compressive Sensing, Spatial-Temporal correlation, Kronecker Product, Data Collection, Energy Efficiency

Abstract: In order to improve the energy efficiency by reducing the amount of the data delivered in Wireless Sensor Networks(WSNs), a Compressive Sensing(CS) based data collection scheme considering the correlation in temporal-spatial domain is studied in this paper. Kronecker product is applied to construct the sparse basis in the joint domain. The simulation results show that due to the huge amount of sensor nodes, by exploiting the dependency in spatial domain, the data number can be reduced distinctly. The high recovery accurancy can still be achieved.

\section{Introduction}

In most cases, communication cost is significant in WSNs. In order to improve the energy efficiency, optimal algorithms[1,3] for the compression of sensed data, communication and sensing have been investigated. In this paper, a CS-based data collection scheme using WSN is designed and simulated to show the improvement of the engergy efficiency.

\section{Compressive Sensing}

To describe CS theory[4,6] concisely, we describe a general linear system with

$$
y=A x .
$$

where $x$ is the target signal in vector form of length $n$, the vector $y$ is the observations with length $m$, and $A$ is a matrix of size $m$ by $n, m \ll n$. If the signal is sparse, Eq. (1) can be rewritten as

$$
y=A \phi C \quad \text { or } \quad y=\psi c .
$$

where $\psi=A \phi$ of size $m \times n$ and $x=\phi c, c$ is a sparse representation when the transform matrix $\phi$ is adopted. In general, $\phi$ is $n \times l$ with $l$ greater or equal to $n$.

CS uses a basis pursuit (BP) approach to find the solution to Eq.(2). For measurements contaminated by noise, BP can be replaced by Basis Pursuit De-Noise (BPDN). The solution is given by

$$
\min \|c\|_{l_{1}} \text { s.t. }\|y-A \phi C\|_{l_{2}} \leq \varepsilon .
$$


Because the $L 1$ norm is convex, it can then be calculated by modern linear programming optimization algorithms.

\section{Model of the Signal in CS-based WSN}

Suppose a WSN consists of N sensor nodes indexed by vector $\tilde{N}=\{1,2, \ldots, N\}$ and a sink node. The samples collected over $T$ sensing periods are represented by a 3-D signal ensemble $\tilde{\mathrm{X}} \in \mathbb{R}^{T \times N_{1} \times N_{2}}$. The $\left(t, n_{1}, n_{2}\right)^{\text {th }}$ sample in ensemble $\tilde{\mathrm{X}}$ denoted as $x_{t, n_{1}, n_{2}}$ refers to the sample in the $t^{\text {th }}$ instant of node $\left(n_{1}, n_{2}\right)$. The equation of $N=N_{1} \times N_{2}$ is here implied. Particularly, the following two expressions of the signal samples are also defined, i.e., $\tilde{\mathrm{X}}_{\left(:, n_{1}, n_{2}\right)} \in \mathbb{R}^{T}$ denotes the vector of $\mathrm{T}$ samples of node $\left(n_{1}, n_{2}\right)$ and $\tilde{\mathrm{X}}_{(t, ;, ;)} \in \mathbb{R}^{N_{1} \times N_{2}}$ denotes the matrix of signal samples at time instant $t=1,2, \ldots, T$. They are merged by the matrix of

$$
\mathrm{X}=\left[x_{1}, x_{2}, \ldots, x_{N}\right]=\left[x^{1}, x^{2}, \ldots, x^{T}\right]^{T} \in \mathbb{R}^{T \times N} .
$$

where $x_{i} \in \mathbb{R}^{T}$ is the $i^{\text {th }}$ column of $\mathrm{X}$ representing the temporal signal of sensor $i \in N$, whereas $x^{t} \in \mathbb{R}^{N}$ is the $t^{t h}$ row representing the spatial WSN signals at time instant $t$. Particularly, $\quad x^{t} \quad$ is formed by stacking $\tilde{\mathrm{X}}_{(t, \ldots ;)}$ into a vector, i.e., $x^{t}=\left[\tilde{X}_{t 11}, \ldots, \tilde{X}_{t 1 N_{2}}, \tilde{X}_{t 21}, \ldots, \tilde{X}_{t 2 N_{2}}, \ldots, \tilde{X}_{t N_{1} N_{2}}\right]^{T}$.

\subsection{CS-Based Signal Model in Temporal Domain}

As assumed above, $x_{i} \in \mathbb{R}^{T}, \quad i=1,2, \ldots, N$ is compressible in temporal domain. According to CS theory, there is a basis $\phi_{T} \in \mathbb{R}^{T \times T}$ such that

$$
x_{i}=\phi_{T} c_{T, i}, i=1,2, \ldots N \text {. }
$$

where $c_{T, i} \in \mathbb{R}^{T}$ is the vector of $k_{i}$-compressible trasform domain coefficients. By denoting $C_{T}=\left[c_{T, 1}, c_{T, 2}, \ldots c_{T, N}\right]$, signal ensemble $X$ with temporal tranformation can be compactly written as $X=\phi_{T} C_{T}$, i.e.,

$$
\left[x_{1}, \ldots, x_{N}\right]=\phi_{T}\left[c_{T, 1}, c_{T, 2}, \ldots c_{T, N}\right] \text {. }
$$

By (2), each sensor $i \in \tilde{N}$ obtains $m_{i}<T$ measurements via measurement matrix $\psi_{i}$ of size $m_{i} \times T$ as

$$
y_{i}=\psi_{i} x_{i}
$$

where $y_{i} \in \mathbb{R}^{m_{i}}$ are the measurements. Then, each sensed data can be recovered according to (3) by solving 


$$
\hat{c}_{T, i}=\arg \min \left\|c_{T, i}\right\|_{l_{1}} \quad \text { s.t. } \quad\left\|y_{i}-\psi_{i} \phi_{T} c_{T, i}\right\|_{l_{2}}<\varepsilon .
$$

then, we can obtain the estimate as $\hat{x}_{i}=\phi_{T} \hat{c}_{T, i}, i=1,2, \ldots N$.

Assume that at instant $t$ only $n_{t}$ sensor nodes are in the active set $A_{t}=\left\{A_{t}(1), \ldots, A_{t}\left(n_{t}\right)\right\}$, $\left|A_{t}\right|=n_{t}$, and accordingly the $A_{t}(n)^{\text {th }}\left(n=1, \ldots, n_{t}\right)$ entries are the available measurements while all the others are zero. Thus, the measurements at instant $t$ can be expressed as $Z^{t}=\left[\tilde{X}_{t A_{t}(1)}, \ldots, \tilde{X}_{t A_{t}\left(n_{t}\right)}\right]^{T} \in \mathbb{R}^{n_{t}} . \quad$ The measurements transmitted in WSN are $\left\{y_{1}, \ldots, y_{N}\right\}=\left\{\psi_{1} x_{1}, \ldots, \psi_{N} x_{N}\right\}$. The total number of the measurements is $M_{T}=\sum_{i=1}^{N} m_{i}$.

\subsection{CS-Based Signal Model in Spatial Domain}

With the assumption of the compressibility of the data sensed in WSN, there are also such basises as $\phi_{1} \in \mathbb{R}^{N_{1} \times N_{1}}$ and $\phi_{2} \in \mathbb{R}^{N_{2} \times N_{2}}$ for vectors $\tilde{\mathrm{X}}_{(t, ; ;)} \in \mathbb{R}^{N_{1} \times N_{2}}$ that they can be expressed by

$$
\tilde{\mathrm{X}}_{(t, ; ;)}=\phi_{1} C_{S, t} \phi_{2}^{T} \quad t=1,2, \ldots T .
$$

where matrix $C_{S, t}$ consists of the associated coefficients in spatial domain.

According to rules of linear algebra, we can express the vector $x^{t}$ as

$$
x^{t}=\operatorname{vec}\left(\tilde{\mathrm{X}}_{(t,:,:)}\right)=\operatorname{vec}\left(\phi_{1} C_{S, t} \phi_{2}^{T}\right)=\left(\phi_{2} \otimes \phi_{1}\right) \operatorname{vec}\left(C_{S, t}\right)=\phi_{S} \operatorname{vec}\left(C_{S, t}\right) \text {. }
$$

where $\phi_{S}=\phi_{2} \otimes \phi_{1} \in \mathbb{R}^{N \times N}$ is the joint spasifying basis for 2-D spatial data, $\otimes$ denotes the Kronecker product and $C_{S, t} \in \mathbb{R}^{N}$ is the vector of $k_{i}$-compressible transform domain coefficients. By denoting $C_{S}=\left[c_{S, 1}, c_{S, 2}, \ldots, c_{S, T}\right]$, the spatial transformation of signal ensemble $X$ is compactly represented as $X^{T}=\phi_{S} C_{S}$, i.e.,

$$
\left[x^{1}, \ldots, x^{T}\right]=\phi_{S}\left[c_{S, 1}, c_{S, 2}, \ldots c_{S, T}\right] \text {. }
$$

Assume we acquire $n_{t}<N$ measurements of the sensor samples at each time instant $t=1,2, \ldots, T$, then also by (2) in CS theory above, we have

$$
z^{t}=\zeta_{t} x^{t}
$$

where $z \in \mathbb{R}^{n_{t}}$ are the measurements, $\zeta_{t} \in \mathbb{R}^{n_{t} \times N}$ is the measurement matrix. According to (3), the recovery of each sensor signal $x^{t}$ for time instant with spatial correlation can be calculated by solving

$$
\hat{c}_{S, t}=\arg \min \left\|c_{S, t}\right\|_{l_{1}} \quad \text { s.t. }\left\|z_{t}-\zeta_{t} \phi_{S} c_{S, t}\right\|_{l_{2}}<\varepsilon .
$$

Then the estimate of $\hat{x^{t}}$ can be further reconstructed as $\hat{x}^{t}=\phi_{S} \hat{c}_{S, t}$. The overall measurements 
delivered in WSN are $\left\{z^{1}, \ldots, z^{T}\right\}=\left\{\zeta_{1} x^{1}, \ldots, \zeta_{T} x^{T}\right\}$. The number of these measurements is $M_{S}=\sum_{t=1}^{T} n_{t}$.

\subsection{CS-Based Signal Model in Spatial-Temporal Domain}

In order to derive the sparsifying basis matrix of the joint correlation structure of $\mathrm{X}$, we combine the basis matrixs above applying the rules of Kronecker product. Assume $\bar{x}$ is the vector-reshaped expression of $\mathrm{X}$ which can be expressed as

$$
\bar{x}=\operatorname{vec}(X)=\left(\phi_{2} \otimes \phi_{1} \otimes \phi_{T}\right) \bar{c}=\left(\phi_{S} \otimes \phi_{T}\right) \bar{c}=\phi_{J} \bar{c} .
$$

where $\bar{x}=\left[\left(x_{1}\right)^{T}, \ldots,\left(x_{N}\right)^{T}\right]^{T} \in \mathbb{R}^{T N}, \phi_{J} \in \mathbb{R}^{T N \times T N}$ is just the equivalent sparsifying basis of the joint correlation structure of $\mathrm{X}, \bar{c} \in \mathbb{R}^{T N}$ is the associated coefficients in transform domain.

For the whole WSN, the measurements $\bar{y}$ can be denoted as

$$
\bar{y}=\psi_{J} \bar{x} .
$$

where $\bar{y}=\left[y_{1}^{T}, \ldots, y_{N}^{T}\right]^{T} \in \mathbb{R}^{\sum m_{i}}$. As assumed above, each sensor $i \in \tilde{N}$ have $m_{i}<T$ measurements on the basis of CS in temporal domain. Recall $y_{i}=\psi_{i} x_{i}$ and $\psi_{i} \in \mathbb{R}^{m_{i} \times T}$, we can deduce $\psi_{J}=\operatorname{diag}\left\{\psi_{1}, \ldots, \psi_{N}\right\} \in \mathbb{R}^{\sum m_{i} \times N T}$. Specifically, if the measurement matrix $\psi_{i}$ of each sensor are identical, i.e., $\psi_{i}=\psi^{\prime}, \quad i \in \tilde{N}$, then (15) can be rewritten as

$$
\bar{y}=\left(I_{N} \otimes \psi^{\prime}\right) \bar{x} \text {. }
$$

where $I_{N}$ is the identity matrix of size $N \times N$.

Continue with the recovery of the signal ensemble for each sensor in WSN, the decoder in sink node can carry it out by solving the following joint optimization based on CS theory as

$$
\hat{\bar{c}}=\arg \min \|\bar{c}\|_{l_{1}} \quad \text { s.t. }\left\|\bar{y}-\psi_{J} \phi_{J} \bar{c}\right\|_{l_{2}}<\varepsilon
$$

resulting in $\hat{\bar{X}}=\phi_{J} \hat{\bar{c}}=\left[\hat{x}^{T}, \ldots, \hat{X}_{N}^{T}\right]^{T}$. Then $\hat{\bar{X}}$ can be further reshaped into the estimate of $\hat{X}=\left[\hat{x_{1}}, \ldots, \hat{x}_{N}\right] \in \mathbb{R}^{T \times N}$.

Data aggregation will be possibly initiated in multi-hop WSN to linearly combine the nodes' measurements. In this case, a measurement matrix $\zeta_{t}^{\text {all }} \in \mathbb{R}^{n_{t} \times N}$ is alternatively selected for (12) involving all the sensors to participate in the measurement process. Each sensor $i \in \tilde{N}$ transmits a CS data packet containing measurements of its own and those from neighbour sensors transmitted via it. The ordered set $F_{i}=\left\{F_{i}(1), \ldots, F_{i}\left(J_{i}\right)\right\}$ collects the neighbour sensors which forward their data via sensor $i$ and $J_{i}$ is the number of the neighbour sensors. Applied the data aggregation 
scheme here, the CS data packet can be denoted as $\left\{x_{t i}, x_{t F_{i}(1)}, \ldots, x_{t F_{i}\left(J_{i}\right)}\right\}$, if $J_{i}<n_{t}$, or $\left\{x_{t i} \omega_{t, i}+\sum_{j \in F_{i}} x_{t j} \omega_{t, j}\right\}$, if $J_{i} \geq n_{t}$, where $\omega_{t, i} \in \mathbb{R}^{n_{t}}$ is the $i^{t h}$ column of $\zeta_{t}^{\text {all }}$ locally generated by sensor $i$. Under the CS-based data aggregation, the maximum number of measurements each sensor node has to transmit is $n_{t}, t=1, \ldots, T$. Then the number of measurements transmitted in network is $M_{\text {all }}=\sum_{t=1}^{T} n_{t} \leq N T$.

\section{Simulation Results}

Assume that the WSN consists of 100 sensor nodes randomly deployed in a square area according to the uniform distribution. In $T=8$ time instants, each sensor collects and transmits samples to the sink node in the center of network. We use i.i.d. Gaussian Matrices for $\psi_{i}$ with $m_{i}=M=20, \forall i \in \tilde{N}$ and $n_{t}=J, \forall t=1, \ldots, T$ respectively. Each sparse basis $\phi_{1}, \phi_{2}$ and $\phi_{T}$ was set to the inverse of DCT-matrix. All the involved optimation questions were solved using BPDN.

The numbers of the measurements sensor nodes have to transimit in WSN are used to compare the communication costs in the case of different signal models, as shown in Fig.1. We define the communication cost ratio as

$$
R_{c}=\sum_{i=1}^{N} N_{i}^{C S} / \sum_{i=1}^{N} N_{i}^{M H F}
$$

where $N_{i}^{C S}$ and $N_{i}^{M H F}$ denote the total numbers of measurements sensor note $i \in \tilde{N}$ has to transmit for the CS-based method and for MHF, respectively. The recovery error is expressed as $\operatorname{NMSE}(d B)=10 \log _{10}\left(E\|\hat{\bar{X}}-\bar{x}\|_{2}^{2} / E\|\bar{x}\|_{2}^{2}\right)$ and the results are plotted in Fig.2.

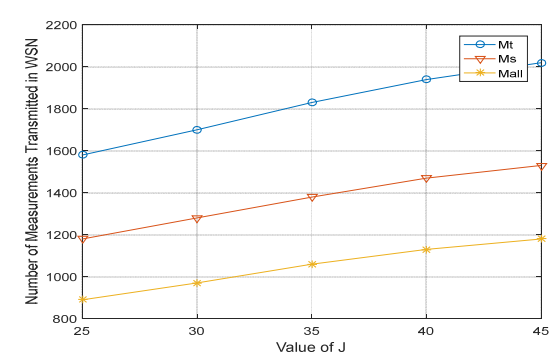

Fig.1 Numbers of measurements transmitted in WSN

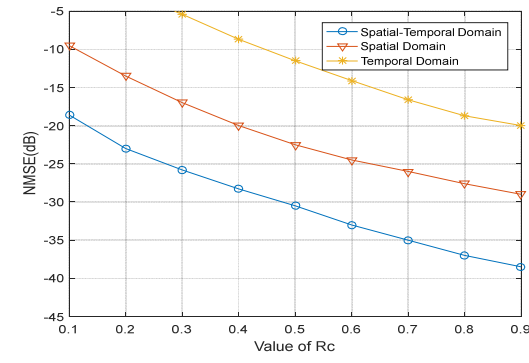

Fig. 2 the Normalized Mean Square Error of each case

\section{Conclusions}

By expoiting the correlatlion in temporal and spatial domains, the number of the measurements is obviously decreased which means the energy consumed by data communication is consequently reduced. Due to the huge amount of the sensors result in high spatial correlation, the energy saving can be efficiently performed with good recovery quality. 


\section{Acknowledgements}

This work was financially supported by the Natural Science Foundation of Gansu Province, China (1506RJZA081).

\section{References}

[1] G.Anastasi, M.Conti, M. Di Francesco and A. Passarella, "Energy conservation in wireless sensor networks: A survey", Ad Hoc Netw, 2009, 7, pp537-568.

[2] Markus Leinonen, Marian Codreanu and Markku Juntti, "Distributed Correlated Data Gathering in WirelessSensor Networks via Compressed Sensing", 2013 Asilomar Conference on Signals, Systems and Computers, Nov. 2013, pp.418-422.

[3] M.A. Razzaque, C. Bleakley and S. Dobson, "Compression in wireless sensor networks: A survey and comparative evaluation", ACM Trans. Sens. Netw. 2013, Vol.10, 5,pp.1-44.

[4] F. Li, T. J. Cornwell and F. de Hoog, "The application of compressive sampling to radio astronomy I: Deconvolution”, Astronomy and Astrophysics, June 2011, Vol.528, A31.

[5] Emmanuel J. Candes, J. Romberg and T. Tao, "Robust uncertainty principles: Exact signal reconstruction from highly incomplete frequency information", IEEE Trans. Inform. Theory, Feb. 2006, vol. 52, no. 2, pp. 489-509.

[6] D. L. Donoho, “Compressed sensing”, IEEE Trans. Inform. Theory, Apr. 2006, vol. 52, no. 4, pp. 1289-1306. 